\title{
On the Modeling and Simulation of Non-Hydrostatic Dam Break Flows
}

\author{
Alexandre Caboussat Sébastien Boyaval \\ Alexandre Masserey
}

January 1, 2013

\begin{abstract}
The numerical simulation of three-dimensional dam break flows is discussed. A non-hydrostatic numerical model for free-surface flows is considered, which is based on the incompressible Navier-Stokes equations coupled with a volume-of-fluid approach. The numerical results obtained for a variety of benchmark problems show the validity of the numerical approach, in comparison with other numerical models, and allow to investigate numerically the non-hydrostatic three-dimensional effects, in particular for the usual test cases where hydrostatic approximations are known analytically. The numerical experiments on actual topographies, in particular the Malpasset dam break and the (hypothetical) break of the Grande-Dixence dam in Switzerland, also illustrate the capabilities of the method for large-scale simulations and real-life visualization. Keywords: Free-surface hydrodynamical flows, Hydraulic engineering, Volume-of-fluid modeling, Three-dimensional non-hydrostatic model, Dam breaks, Finite-element method.
\end{abstract}

\section{Introduction and Motivations}

One essential feature of dam break studies consists in accurately forecasting the fast floods that are incurred in the area (valleys) below a dam by the failure of the dam structure. The determination of the potential consequences of a dam break requires the spatial location of the flood, as well as the time evolution of the flow in terms of fronts speed and water height.

Physical models have been used for a long time to predict the impacts of dam breaks, but they are costly and not always accurate enough because of the limited measurability of some quantities and a scale that is smaller than real-life situations.

Numerical models on the contrary have now proved quite accurate and of reasonable cost in a number of studies, see the numerous references below. Furthermore, in comparison with physical models, they can provide details at any 
point of the flow. Though, a careful and accurate validation of numerical solutions remains difficult. On the one hand, because of an uncomplete mathematical theory, and on the other hand, because of computational time and memory limitations, in particular as concerns the full 3D models that compute numerical approximations of the solutions to the non-hydrostatic ("full") threedimensional (3D) Navier-Stokes equations with free-surface (and possibly open) boundary conditions.

In fact, the question of the design of an accurate simplified model dedicated to the dam break problem is recurrent in the literature, see, e.g., [4, 17, 18, 33]. Several simplified models with a much more reasonable cost than full 3D models have indeed succeeded in exhibiting numerical results that are in adequation with experimental results, see, e.g., [1]. But the simplified models are inherently biased, and the accurate quantification of the model error compared with a full 3D model remains a well-known mathematical challenge. Simplified models are mainly hydrostatic models $[1,18,35]$, possibly with only a one-dimensional description of the front propagation, while the non-hydrostatic effects become not negligible over rough topographies e.g. in dam break flows ${ }^{1}$, let alone $3 \mathrm{D}$ effects.

Since it has recently become possible to carry out 3D numerical simulations, see e.g. $[3,9,10,21,29,36]$, the goal of this article is thus two-fold, with a view to overcoming some of the modeling errors that necessarily arise in simplified models [23, 26, 27].

First, to highlight the consequences of non-hydrostatic, three-dimensional effects (through computer analyses), we discuss numerical solutions to the full 3D Navier-Stokes equations with free-surface boundary conditions for the usual benchmark problems where simplified hydrostatic 1D flows are known exactly and which have been used extensively in the past for validating the specific simulation of dam break flows. Indeed, without a complete mathematical theory, but with a view to building it, we believe it useful to numerically investigate the essential features of full 3D models in simple benchmark situations. Generalization is achieved via some classical test cases such as the asymmetric dam breach or the constriction of a flow in a channel, in order to highlight non-hydrostatic effects.

Second, we illustrate the interest of large-scale real-life simulations with that full 3D model for the industry and land-planners. It seems indeed desirable, not only for an accurate mathematical understanding, but also for a fast and systematic planning procedure, that generic (full, 3D) numerical models can easily reproduce correctly the essential features of a dam break flows without any tuning or parametrization in most situations. The introduction of additional physical features requiring the tuning of parameters, such as friction boundary conditions or turbulence models, could be considered in future works. Such parametrized dissipation models are useful when the numerical diffusion is too

\footnotetext{
${ }^{1}$ Formally, non-hydrostatic effects are expected to be small compared to the hydrostatic mainstream approximation, provided the bottom topography is flat enough [15]. The hydrostaticity assumption is also famously not adequate when trying to reproduce some surface waves [5].
} 
small compared to the physical one. However, numerical results show that it is not a problem for the test cases considered in this work. Since 3D models are naturally more dissipative than reduced $1 \mathrm{D}$ or $2 \mathrm{D}$ models, it is actually remarkable to observe that our numerical results are comparable to those obtained with reduced models.

The article is organized as follows. In the next section, the mathematical and physical models are presented. The numerical algorithms are then briefly described. (The numerical method presented in this article for the full 3D numerical simulation of dam break flows is based on a Volume-Of-Fluid (VOF) modeling of free surfaces and has already been used in the past for several situations where free-surface flows occur $[6,8,25]$.) Numerical experiments are illustrated in the last section.

Benchmark problems have been considered first in order to validate the numerical simulation of flooding waves (simple dam break flows where data for approximations of the water height and mean velocity are available, either analytical expressions that are exact solutions to simplified models or well-documented numerical solutions). The method is numerically demonstrated to converge in test cases that are exactly solvable for the well-known hydrostatic model based on the inviscid Saint-Venant equations for shallow water (the Ritter test case [30] and the Stoker test case [34], where the velocity is one-dimensional). The same 3D non-hydrostatic features as in [15] appear, even if small, in a thin-layer regime when the Navier-Stokes equations formally reduce to the Saint-Venant equations. This is a clear manifestation of the modeling error.

We also compare with numerical solutions to reduced shallow-water models in a well-documented test case $[4,12,35]$ that consists of a simplified dam breach over a wet bed (a 3D asymmetric extension of the Stoker test case). Non-hydrostatic 3D features similar to the previous test cases also exist.

Last, we tackle two real-life situations in large geometrical domains (up to 20000 [m] long). The test cases use real topologies and are computational challenges when using a 3D approach due to the large scales. We insist on the fact that the real topographies are handled naturally without any parametrization, contrary to most simplified models where a non-smooth topography implies difficulties both on the numerical and modelling viewpoints. In addition to show the capabilities of our numerical method, such simulations also show that realistic results can be achieved with a model using as few parameters as possible: we use neither friction nor turbulence model (and thus do not tune any physical or numerical parameters).

The first example is the Malpasset dam break, which has been used extensively in the past for the validation of numerical models, see e.g. [2, 14, 17, 32]. To the best of our knowledge, only qualitative results of the Malpasset dam break have been presented when using numerical simulation in three dimensions. Here, we aim at quantifying the three-dimensional approach. The final example (Grande-Dixence dam break) illustrates the potentialities of the numerical approach in real topographies and large computational domains, in particular for policy makers to forecast floods and protect cities and inhabitants.

The Malpasset test case allows to discuss the importance of the non-flat 
bottom on the non-hydrostatic features of the flow. The Grande-Dixence test case shows the potential importance of such simulations for disaster predictions, together with the need for an appropriate visualization of the simulation for industrial partners.

Finally, our results show that, although 3D numerical simulations remain computationally very expensive, so simplified models in turn remain useful for a number of "real-time" and "optimization" purposes in particular, full 3D models should definitely be used, either to help designing appropriate simplified models in specific situations that remain an actual challenge to model reduction, or at least as benchmarks to be compared with various simplified models as long as they are practicable.

\section{A VOF Approach to Free Surfaces Flows}

Let us define $T>0$ as the final time of simulation and consider a bounded computational domain $\Lambda \subset \mathbb{R}^{3}$ in which the fluid remains confined for all times $t \in[0, T]$. The domain actually occupied by the water at any given time $t$ is denoted by $\Omega_{t} \subset \Lambda$ and the free surface between the water and the air by $\Gamma_{t}=\partial \Omega_{t} \backslash \partial \Lambda$. Let $Q_{T}$ denote the space-time domain containing the water, that is $Q_{T}=\left\{(\mathbf{x}, t): \mathbf{x} \in \Omega_{t}, 0<t<T\right\}$. The velocity field $\mathbf{v}: Q_{T} \rightarrow \mathbb{R}^{d}$ and the pressure field $p: Q_{T} \rightarrow \mathbb{R}$ shall satisfy the incompressible Navier-Stokes equations in $Q_{T}$ :

$$
\begin{aligned}
& \rho \frac{\partial \mathbf{v}}{\partial t}+\rho(\mathbf{v} \cdot \nabla) \mathbf{v}-2 \nabla \cdot(\mu \mathbf{D}(\mathbf{v}))+\nabla p=\mathbf{f} \\
& \nabla \cdot \mathbf{v}=0
\end{aligned}
$$

where $\mathbf{D}(\mathbf{v})=1 / 2\left(\nabla \mathbf{v}+\nabla \mathbf{v}^{T}\right)$ is the symmetric deformation rate tensor, $\rho$ the constant density of water, $\mu$ the constant molecular viscosity of the water and $\mathbf{f}$ denotes the external forces (that is the gravitational forces $\mathbf{f}=\rho \mathbf{g}$ here, with $\mathbf{g}$ the gravity acceleration vector). At any given time $t$, slip or no-slip boundary conditions are enforced on the boundary of the water domain $\Omega_{t}$ that is in contact with the boundary of the computational domain viz. $\partial \Lambda \cap \partial \Omega_{t}$. On the water-air interface $\Gamma_{t}$, we require free-surface forces:

$$
-p \mathbf{n}+2 \mu \mathbf{D}(\mathbf{v}) \mathbf{n}=0 \quad \text { on } \Gamma_{t}, \quad t \in(0, T),
$$

where $\mathbf{n}$ is the unit normal of the water-air free surface oriented toward the air domain.

With a view to numerical computation, we model the free-surface following the VOF approach [25]. The position of the water at time $t$ is tracked by a characteristic function $\varphi: \Lambda \times(0, T) \rightarrow \mathbb{R}$. The function $\varphi$ equals one if water is present, zero if it is not. Initial conditions are given for $\varphi$ to define the initial water region $\Omega_{0}=\{\mathbf{x} \in \Lambda: \varphi(\mathbf{x}, 0)=1\}$, as well as for the velocity field $\mathbf{v}$ (initially prescribed in $\Omega_{0}$ ). The kinematics of the free surface is that of a 
material surface so that $\varphi$ satisfies

$$
\frac{\partial \varphi}{\partial t}+\mathbf{v} \cdot \nabla \varphi=0 \quad \text { in } \Lambda \times(0, T),
$$

where $\mathbf{v}$ outside $Q_{T}$ can be any continuous extension of $\mathbf{v}$ inside $Q_{T}$. (Note that $\varphi(\mathbf{X}(t), t)=\varphi(\mathbf{X}(0), 0)$ is uniquely defined whenever the trajectories $\mathbf{X}(t)$ of fluid particles at position $\mathbf{X}(0)$ at time $t=0$, thus such that $\mathbf{X}^{\prime}(t)=\mathbf{v}(\mathbf{X}(t), t)$, do not collide.)

For future reference, let us briefly recall how (1)-(4) can be formally reduced to a shallow-water model (see e.g. $[15,24]$ for more details). The goal of the model reduction is to derive a closed set of equations simpler than (1)(4) for (approximations of) $\mathbf{v}$ and $\varphi$, when the free surface is supposed to be "non-folded" over a similarly non-folded topography. That is, assuming $\Lambda=\{(x, y, z) \in S \times(0, Z)\}$ is a cylinder with base $S \subset \mathbb{R}^{2}$ and axis directed along the gravity acceleration vector, the free surface is required to be a piecewise smooth manifold with equation $z=b(x, y)+\bar{h}(t, x, y)$ while $z=b(x, y)$ is the given topography equation (think of rivers and lakes). Using scaling assumptions in a thin-layer regime with slip boundary conditions on a slowly varying topography $(|\nabla b| \ll 1)$, one can show formally that $\mathbf{v} \approx(u, v, 0)$ and $p \approx \rho g \bar{h}$ where $(\bar{h}, u, v)$ satisfy the viscous Saint-Venant equations in $S \times(0, T)$ :

$$
\begin{aligned}
& \frac{\partial \bar{h}}{\partial t}+\frac{\partial \bar{h} u}{\partial x}+\frac{\partial \bar{h} v}{\partial y}=0, \\
& \bar{h} \frac{\partial u}{\partial t}+\bar{h} u \frac{\partial u}{\partial x}+\bar{h} v \frac{\partial u}{\partial y}+g \bar{h} \frac{\partial \bar{h}}{\partial x}=k_{x}-g \bar{h} \frac{\partial b}{\partial x} \\
& -\frac{\mu}{\rho}\left(\frac{\partial}{\partial x}\left(\bar{h}\left[3 \frac{\partial u}{\partial x}+\frac{\partial v}{\partial y}\right]\right)+\frac{\partial}{\partial y}\left(\bar{h} \frac{\partial v}{\partial x}+\bar{h} \frac{\partial u}{\partial y}\right)\right), \\
& \bar{h} \frac{\partial v}{\partial t}+\bar{h} u \frac{\partial v}{\partial x}+\bar{h} v \frac{\partial v}{\partial y}+g \bar{h} \frac{\partial \bar{h}}{\partial x}=k_{y}-g \bar{h} \frac{\partial b}{\partial y} \\
& -\frac{\mu}{\rho}\left(\frac{\partial}{\partial y}\left(\bar{h}\left[\frac{\partial u}{\partial x}+3 \frac{\partial v}{\partial y}\right]\right)+\frac{\partial}{\partial x}\left(\bar{h} \frac{\partial u}{\partial y}+\bar{h} \frac{\partial v}{\partial x}\right)\right) .
\end{aligned}
$$

Considering the smaller number of unknowns, it is a priori computationally less costly to solve the Saint-Venant system (5)-(7) than the full 3D NavierStokes equations. Note that the viscous terms in (5)-(7) are very small for water $\left(\rho|\mathbf{v}||\Lambda|^{1 / 3} / \mu \gg 1\right)$ and occur only in the "second-order" approximation to (1)-(4), but their importance arises through the determination of a unique entropic solution to the inviscid Saint-Venant system of balance laws obtained in the vanishing viscosity limit $\frac{\mu}{\rho} \rightarrow 0$, recall, e.g., $[15,24]$. (At the numerical level, one can either solve a variational formulation of (5)-(7) like [21], or more commonly capture entropic solutions to the inviscid limit of (5)-(7) and next add viscous perturbations like, e.g., [32].) The force term $\left(f_{x}, f_{y}\right)$ is typically parametrized to account for friction and turbulence at the bottom topography.

The main limitations of the reduced model are a hydrostatic pressure, which is consistent with small vertical velocities and a horizontal motion "by slices" (where a given velocity profile is imposed all along horizontal directions), and a non-folded description of the free surface, which is consistent with a nonbreaking flow attached to the topography. 
Although the latter consistency assumptions seems reasonable in a number of applications to geophysical flows, which explains why the shallow-water reduced model has proved useful to many applications in hydraulics (see the discussion about dam break flows in Section 1), they are not satisfied in general and may locally induce strong inaccuracies, in particular where the bottom topography $b$ varies fast. Then, a full $3 \mathrm{D}$ modeling of dam break flows may be useful, at least for benchmarking purposes.

\section{Numerical Discretization}

The advocated numerical algorithm relies on a time splitting method to decouple advection and diffusion phenomena and a two-grid approach for the space discretization. It is straightforwardly adapted from [25] and only briefly described hereafter. We recall that one goal of this work is to validate the method for dam break simulations.

\subsection{Time Splitting Scheme}

Let $\tau>0$ be a given time step and $t^{n}=n \tau, n \geq 0$, be a sequence of discrete times. Let $\varphi^{n}, \mathbf{v}^{n}, \Omega^{n}$ be approximations of $\varphi, \mathbf{v}, \Omega$ respectively at time $t^{n}$. The approximations $\varphi^{n+1}, \mathbf{v}^{n+1}, \Omega^{n+1}$ at time $t^{n+1}$ are computed by a splitting algorithm illustrated in Figure 1.

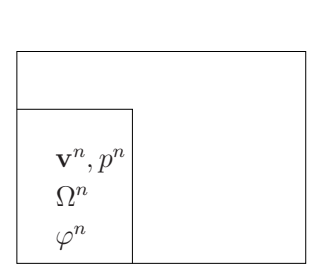

Time $t^{n}$

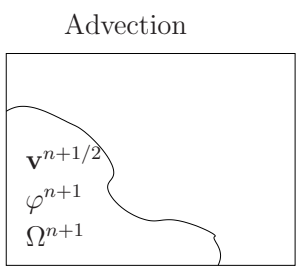

$$
\text { Time } t^{n+1}
$$

Figure 1: The splitting algorithm (from left to right) for a dam break flow (collapse of a column of water initially located on the left of the domain). Two advection problems are solved to determine the new approximation of the characteristic function $\varphi^{n+1}$, the new liquid domain $\Omega^{n+1}$ and the predicted velocity $\mathbf{v}^{n+1 / 2}$. Then, a time dependent Stokes problem is solved to obtain the velocity $\mathbf{v}^{n+1}$ and the pressure $p^{n+1}$ in the new liquid domain $\Omega^{n+1}$.

First two advection problems are solved, that lead to the new approximation of the characteristic function $\varphi^{n+1}$ together with a prediction $\mathbf{v}^{n+1 / 2}$ of the new velocity in the new water domain $\Omega^{n+1}$ with free surface $\Gamma^{n+1}$. (The domain $\Omega^{n+1}$ is defined as the set of points such that $\varphi^{n+1}$ equals one.) This advection step consists in solving, with an (explicit) forward characteristics method, four 
transport equations between $t^{n}$ and $t^{n+1}$ :

$$
\frac{\partial \varphi}{\partial t}+\mathbf{v} \cdot \nabla \varphi=0, \quad \frac{\partial \mathbf{v}}{\partial t}+(\mathbf{v} \cdot \nabla) \mathbf{v}=0
$$

with initial conditions given by the values of the functions $\varphi$ and $\mathbf{v}$ at time $t^{n}$. The solutions read $\varphi^{n+1}\left(\mathbf{x}+\tau \mathbf{v}^{n}(\mathbf{x})\right)=\varphi^{n}(\mathbf{x})$ and $\mathbf{v}^{n+1 / 2}\left(\mathbf{x}+\tau \mathbf{v}^{n}(\mathbf{x})\right)=\mathbf{v}^{n}(\mathbf{x})$ for all $\mathbf{x} \in \Omega^{n}$.

Then, a time dependent Stokes problem is solved in $\Omega^{n+1} \times\left(t^{n}, t^{n+1}\right)$ using the predicted velocity $\mathbf{v}^{n+1 / 2}$ as initial condition. We use an (implicit) backward Euler scheme. The velocity $\mathbf{v}^{n+1}$ and the pressure $p^{n+1}$ are thus solution to a boundary value problem in $\Omega^{n+1}$ :

$$
\begin{aligned}
& \rho \frac{\mathbf{v}^{n+1}-\mathbf{v}^{n+1 / 2}}{\tau}-2 \nabla \cdot\left(\mu \mathbf{D}\left(\mathbf{v}^{n+1}\right)\right)+\nabla p^{n+1}=\mathbf{f}^{n+1}, \\
& \nabla \cdot \mathbf{v}^{n+1}=0,
\end{aligned}
$$

with boundary condition (4) on $\Gamma^{n+1}$ and slip or no-slip elsewhere (recall Section 2).

Two different spatial discretizations are used for the advection problems and for the Stokes problem, as illustrated in Figure 2. A regular grid of square cells $\mathcal{C}_{h}$ is used to solve the advection problems, while the Stokes problem is solved on a coarser unstructured tetrahedral finite element mesh $\mathcal{T}_{H}$.

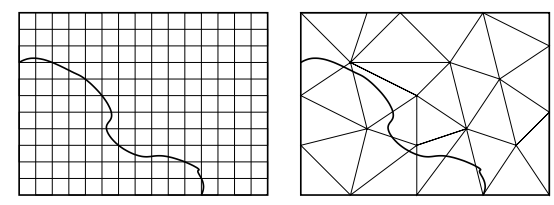

Figure 2: Two-grid method in the two-dimensional case for a dam break flow (collapse of a column of water initially located on the left of the domain): structured grid of small square cells $\mathcal{C}_{h}$ (left) and unstructured finite element mesh of triangles $\mathcal{T}_{H}$ (right).

\subsection{Advection Step}

The cavity $\Lambda$ is embedded into a box that is meshed into a structured grid denoted by $\mathcal{C}_{h}$, made of cubic cells of size $h$. We label each cell $C_{i j k}$ by the multi-index $(i j k)$, where the indices $i, j, k$ vary respectively only in each one of the three spatial directions of a Cartesian frame. All advection steps are solved on the same structured grid $\mathcal{C}_{h}$ with a forward characteristics method detailed in [25], using, at any time $t^{n}$, piecewise constant approximations of $\varphi^{n}$ and $\mathbf{v}^{n}$ on $\mathcal{C}_{h}$ (hence a collection of values $\varphi_{i j k}^{n}, \mathbf{v}_{i j k}^{n}$ indexed by their cell label $(i j k)$ ). The algorithm consists in moving the cell $(i j k)$ in the direction $\tau \mathbf{v}_{i j k}^{n}$ and next 
conservatively distributing the transported quantities $\varphi_{i j k}^{n}$ and $\mathbf{v}_{i j k}^{n}$ into the overlapped cells (with ratio the area intersected by the transported cell divided by the area of the transported cell). Yet, the repeated projection of a cell onto the structured grid is an overly diffusive procedure for accurate propagation of a front like $\Gamma^{n}$.

We use a variation of the heuristic SLIC algorithm developed in [25] and inspired by [28] to reduce the numerical diffusion of the front. The cells where $\varphi_{i j k}^{n}=1$ are advected first. Next, in the cells $(i j k)$ where $0<\varphi_{i j k}^{n}<1$, a square subcell is defined within $(i j k)$, with center such that some of its edges coincide with an edge of the cell $(i j k)$, on the other side of which the approximation of $\varphi^{n}$ is also non-uniformly 0 , if possible. After pushing this way the fluid along the faces of the cell, the subsequent translation and the projection of those latter subcells follow the same characteristic method as for completely filled cells. Once advected, the corresponding quantity is redistributed to the underlying cells of $\mathcal{C}_{h}$ proportionally to the volume of the intersection. The cell advection and projection with SLIC algorithm are presented in Figure 3, for a two-dimensional grid $\mathcal{C}_{h}$ for the sake of simplicity.
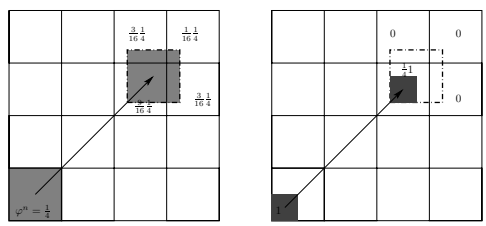

Figure 3: Effect of the SLIC algorithm on numerical diffusion. An example of two dimensional advection and projection when the volume fraction of liquid in the cell is $\varphi_{i j}^{n}=\frac{1}{4}$. Left: without SLIC, the volume fraction of liquid is advected and projected on four cells, with contributions (from the top left cell to the bottom right cell) $\frac{3}{16} \frac{1}{4}, \frac{1}{16} \frac{1}{4}, \frac{9}{16} \frac{1}{4}, \frac{3}{16} \frac{1}{4}$. Right: with SLIC, the volume fraction of liquid is first pushed at one corner, then it is advected and projected on one cell only, with contribution $\frac{1}{4}$.

Last, to avoid $\varphi_{i j k}^{n+1}>1$ for some $(i j k)$, a post-processing technique redistributes the excess of water from over-filled cells to cells $(i j k)$ where $0<\varphi_{i j k}^{n}<$ 1. Related to global repair algorithms [31], this technique produces final values $\varphi_{i j k}^{n+1}$ which are between zero and one, even when the advection of $\varphi^{n}$ gives values strictly larger than one. The technique consists in moving the fraction of liquid in excess in the cells that are over-filled to receiver cells in a global manner by sorting the cells according to $\varphi^{n+1}$. Details can be found in [25]. In most of our computations, only a small amount of excess water cannot be redistributed (the so-called numerical compression in [25]). 


\subsection{Diffusion Step}

Let $\mathcal{T}_{H}$ be a tetrahedral discretization of the cavity $\Lambda$ satisfying the usual compatibility conditions between tetrahedra to define a FE mesh (see e.g. [16]). The maximal diameter of the elements is denoted by $H$ (typically $H$ is of the order $5 h$ to $10 h)$. We denote by $P_{\ell}$ the nodes of the FE mesh.

Once values $\varphi_{i j k}^{n+1}$ and $\mathbf{v}_{i j k}^{n+1 / 2}$ have been computed on $\mathcal{C}_{h}$, one computes continuous piecewise linear approximations of $\varphi_{H}^{n+1}$ and $\mathbf{v}_{H}^{n+1 / 2}$ on $\mathcal{T}_{H}$ by assigning to each mesh node $P_{\ell}$ an average value in a patch of neighboring cells (a local $L^{2}$-projection with mass lumping):

$$
\begin{aligned}
\varphi_{P_{\ell}}^{n+1} & =\sum_{K \in \mathcal{T}_{H}, K \ni P_{\ell}} \sum_{i j k, C_{i j k} \subset K} \alpha_{i j k \ell} \varphi_{i j k}^{n+1}, \\
\text { with } & \psi_{P_{\ell}}\left(C_{i j k}\right) \\
\alpha_{i j k \ell} & =\frac{\sum_{K \in \mathcal{T}_{H}, K \ni P_{\ell}} i^{\prime} j^{\prime} k^{\prime}, C_{i^{\prime} j^{\prime} k^{\prime}} \subset K}{\psi_{P_{\ell}}\left(C_{i^{\prime} j^{\prime} k^{\prime}}\right)},
\end{aligned}
$$

where $\psi_{P_{\ell}}, \ell=1, \ldots, N$ denotes the continuous piecewise linear functions defining a FE basis with non-zero value only at node $P_{\ell}$. Here $K$ denotes an element (tetrahedron) of the finite element mesh $\mathcal{T}_{H}$, and $N$ denotes the total number of vertices of $\mathcal{T}_{H}$. The notation $P K \ni_{\ell}$ means that the node $P_{\ell}$ is one of the vertices of the element (tetrahedron) $K$. The notation $C_{i j k} \subset K$ means that the center of mass of the cell $C_{i j k}$ is located in the finite element $K$. The same kind of formula is used to obtain the values of the predicted velocity $\mathbf{v}^{n+1 / 2}$ at the vertices $P_{\ell}$. Moreover, note that there is no need to interpolate the pressure on the grid $\mathcal{C}_{h}$ as it does not appear in the advection problems.

We denote by $\Omega_{H}^{n+1}$ the approximation of the water domain $\Omega^{n+1}$ defined as the union of all elements of the finite element mesh such that one of their vertices $P$ has a value $\varphi_{P}^{n+1}>0.5$, and by $\Gamma_{H}^{n+1}$ the approximation of the waterair interface $\Gamma^{n+1}$, thus an error of order $\mathcal{O}(H)$ (an adaptive mesh algorithm for the improvement of that projection error has been discussed in [7].)

Let us denote by $\mathbf{v}_{H}^{n+1}$ (resp. $p_{H}^{n+1}$ ) the piecewise linear approximation of $\mathbf{v}^{n+1}$ (resp. $\left.p^{n+1}\right)$. The Stokes problem is solved with a stabilized discrete variational formulation (Galerkin Least Squares method) and consists in finding the velocity $\mathbf{v}_{H}^{n+1}$ and pressure $p_{H}^{n+1}$ such that:

$$
\begin{aligned}
& \int_{\Omega_{H}^{n+1}} \frac{\mathbf{v}_{H}^{n+1}-\mathbf{v}_{H}^{n+1 / 2}}{\tau} \cdot \mathbf{w} d \mathbf{x}+2 \mu \int_{\Omega_{H}^{n+1}} \mathbf{D}\left(\mathbf{v}_{H}^{n+1}\right): \mathbf{D}(\mathbf{w}) d \mathbf{x} \\
& -\int_{\Omega_{H}^{n+1}} \mathbf{f}^{n+1} \cdot \mathbf{w} d \mathbf{x}-\int_{\Omega_{H}^{n+1}} p_{H}^{n+1} \nabla \cdot \mathbf{w} d \mathbf{x}-\int_{\Omega_{H}^{n+1}} q \nabla \cdot \mathbf{v}_{H}^{n+1} q d \mathbf{x} \\
& -\sum_{K \subset \Omega_{H}^{n+1}} \alpha_{K} \int_{K}\left(\frac{\mathbf{v}_{H}^{n+1}-\mathbf{v}_{H}^{n+1 / 2}}{\tau}+\nabla p_{H}^{n+1}-\mathbf{f}^{n+1}\right) \cdot \nabla q d \mathbf{x} \\
& =0,
\end{aligned}
$$


for all $\mathbf{w}$ and $q$, the velocity and pressure test functions compatible with the boundary conditions on $\partial \Lambda$. The value of the stabilization parameter $\alpha_{K}$ (a function of the local Reynolds number) has been discussed in [25]. The corresponding linear system is solved with a standard GMRES method without restarting technique (from the library SparseLib++), with an ILU preconditioner.

The continuous piecewise linear approximation of the velocity $\mathbf{v}_{H}^{n+1}$ on $\mathcal{T}_{H}$ is finally restricted at the center of each cell $C_{i j k}$ to obtain the values $\mathbf{v}_{i j k}^{n+1}$ on the structured grid $\mathcal{C}_{h}$ for the next advection step. This is an interpolation from the finite elements to the grid of small cells that is the reverse of $(10)(11)$. When the center of mass of the cell $C_{i j k}$ belongs to the element $K$, the new velocity is given by the linear interpolation, based on the finite element basis functions:

$$
\mathbf{v}_{i j k}^{n+1}=\sum_{P_{\ell} \in K} \mathbf{v}_{P_{\ell}}^{n+1} \psi_{P_{\ell}}\left(C_{i j k}\right) .
$$

\section{Numerical Experiments}

The results of several numerical experiments are presented in this section. We present first the classical RitTer [30] and STOKER [34] test cases, when a onedimensional (1D) dam breaks over a dry or wet bed respectively. Since the proposed numerical method has already been validated in the past for freesurface flows, see e.g. [25], the goal of our first examples is to evaluate the impact of a full 3D model compared with a reduced 1D hydrostatic model in standard benchmarks for dam break flows. In those cases, the water level and the depth-averaged velocity solution to the Navier-Stokes equations can be approximated by the solution to the inviscid Saint-Venant equations (5)-(7), a simplified 1D model for shallow-water flows whose solution is analytically known here. Numerical convergence is observed toward a solution close to the analytical formula, as expected for high Reynolds numbers and flat topographies. But (admittedly small) 3D non-hydrostatic effects are also clearly characterized and we briefly discuss them via our computer analyses. In a second step, comparisons with well-documented examples are presented (asymmetric dam, Malpasset dam break). Again, our numerical results are close to the numerous (numerical and physical) data available in the literature, but also show some distinctive features of the 3D non-hydrostatic effects. We end this section with the new real-life simulation of the (hypothetical) break of a large dam in Switzerland, to show how large-scale numerical results can be coupled with efficient visualization techniques. All computations in this Section are achieved on an Intel Xeon $(2.93 \mathrm{GHz})$ with $8 \mathrm{~GB}$ memory.

\subsection{The Ritter Test Case}

The Ritter test case consists of a 1D channel with a flat horizontal bottom (oriented along $O x$ ). The dam is vertical and breaks instantaneously at initial time. The initial conditions for the water height are: 


$$
h_{0}(x)= \begin{cases}h_{0}, & x<a \\ 0, & x \geq a\end{cases}
$$

with zero initial velocity; $h_{0}$ is the initial height of the water and $a$ is the initial location of the dam. Relationship (13) implies that, downstream of the dam, the domain is assumed to be dry, while the fluid upstream is initially at rest. This is a Riemann problem for the inviscid Saint-Venant equations $\left(\frac{\mu}{\rho}=0\right)$ and one exact solution is a rarefaction wave, see, e.g., [11], with fan:

$$
\begin{aligned}
& \bar{h}(x, t)=\frac{\left(2 \sqrt{g h_{0}}-\frac{x-a}{t}\right)^{2}}{9 g}, \\
& u(x, t)=\frac{2}{3}\left(\sqrt{g h_{0}}+\frac{x-a}{t}\right),
\end{aligned}
$$

if $-1<x /\left(t \sqrt{g h_{0}}\right)<2$ (elsewhere, the solution is equal to the initial condition). Numerical simulations can be done using Navier-Stokes equations and a $3 \mathrm{D}$ velocity in the same setting after extruding the computational domain into a transverse direction (hence in a pseudo 2D configuration consisting of a channel with a rectangular cross-section).

We consider the geometry described in [15], namely a channel defined by the domain $(-50,50) \times(0,2) \times(0,3)$. The initial height is $h_{0}=2[\mathrm{~m}]$ and the dam is initially located at abscissa $a=0[\mathrm{~m}]$. The liquid properties are $\mu=10^{-3}[\mathrm{~kg} /(\mathrm{ms})]$ and $\rho=10^{3}\left[\mathrm{~kg} / \mathrm{m}^{3}\right]$ (water). Slip boundary conditions are imposed at the bottom of the channel. The computational cost of the numerical simulations is between 10 minutes $\left(h=8 \cdot 10^{-2}, H=3 / 5, \tau=0.1\right)$, to 12 hours ( $\left.h=4 \cdot 10^{-2}, H=3 / 14, \tau=0.025\right)$. As the mesh size and time step are divided by two (in each direction), one can see that the numerical algorithms scale appropriately.

Figure 4 visualizes the profile of the numerical solution (water height) after reconstruction on several FE meshes (coarse to fine) and its comparison with (14) at times $t=0,3$ and $5[\mathrm{~s}]$. (The CFL number is smaller than one.) One can see that the numerical simulations converge to a Navier-Stokes solution close to the rarefaction wave predicted by the Saint-Venant equations. Yet, differences between the Navier-Stokes and the Saint-Venant predictions are localized close to regions where the derivatives are discontinuous. First, Navier-Stokes equations tend to regularize this singular behavior. Second, the Navier-Stokes front speed seems to slightly slow down as time goes on, in comparison with the Saint-Venant prediction. Of course, this sounds natural in a simulation where the kinetic energy is dissipated because of viscous effects. But this is especially true at the front of the wave. Note indeed that the front shapes are slightly different close to the vacuum. This is not a pure artifact due to the reconstruction on the $\mathrm{FE}$ mesh, insofar as the wave tip decreases as the mesh is refined, but also a natural effect at a point where pure-slip and no surface tension boundary conditions should match. So viscous dissipation and 3D geometry effects especially affect the tip of the wave, where a "thin-layer" approximation has no meaning 
(since $\partial u / \partial x$ jumps). Then, the inertial terms in the momentum balance projected along the gravity direction may not be negligible, a local manifestation of the 3D non-hydrostatic effects, and the limit angle of the free surface at the tip of the wave may not be zero (contrary to the hydrostatic case).
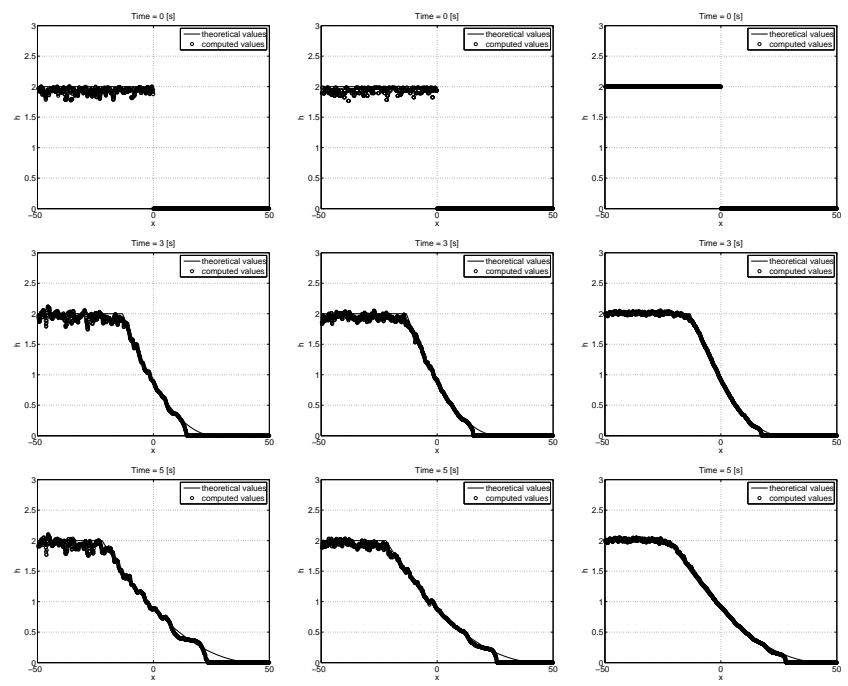

Figure 4: Ritter analytical test case: comparison with analytical solution at times $t=0,3$ and $5[\mathrm{~s}]$. Left: coarse mesh $\left(h=8 \cdot 10^{-2}, H=3 / 5, \tau=0.1\right)$; middle: middle mesh $\left(h=6 \cdot 10^{-2}, H=3 / 8, \tau=0.05\right)$; right: fine mesh $\left(h=4 \cdot 10^{-2}, H=3 / 14, \tau=0.025\right)$. All the grid points on the free surface are represented, including those in the transverse direction.

\subsection{The Stoker Test Case}

In the Stoker test case, the water released as the dam breaks flows over a wet bed instead of a dry bed. We consider the same channel and the same fluid properties as in the previous section, with initial conditions

$$
h_{0}(x)= \begin{cases}h_{l}, & x<a \\ h_{r}, & x \geq a\end{cases}
$$

for the water height, together with zero initial velocity; $h_{l}$ and $h_{r}$ are the initial heights of the water on the left and right sides of the dam, and $a$ is the initial location of the dam on the $O x$ direction. Stoker's exact solution to the inviscid Saint-Venant equations is the superimposition of two waves (see, e.g., [21]): one up-going rarefaction wave and one down-going shock wave connected by a medial zone with a constant depth $h_{m}$ and constant velocity $u_{m}$. Let us define $c_{l}=\sqrt{g h_{l}}, c_{r}=\sqrt{g h_{r}}$ and $c_{m}=\sqrt{g h_{m}}$, then analytical formulas read, 

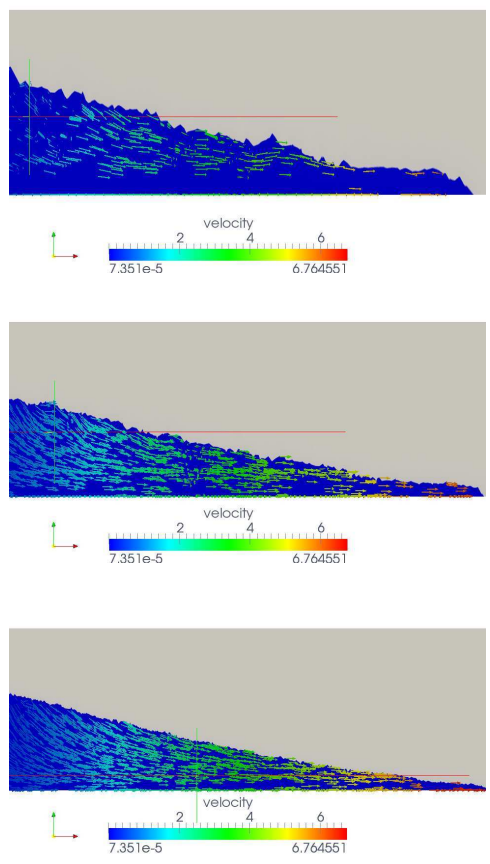

Figure 5: Ritter analytical test case: Visualization of the velocities at time $t=5[\mathrm{~s}]$ next to the liquid front. Top: coarse mesh; middle: middle mesh; bottom: fine mesh. Results magnified by a factor 5 in the vertical direction.

$$
\bar{h}(x, t)= \begin{cases}\frac{h_{l}}{\left(2 \sqrt{g h_{l}}-\frac{x-a}{t}\right)^{2}}, & \text { if } x-a<-c_{l} t, \\ h_{m} & \text { if }-c_{l} t<x-a<\left(u_{m}-c_{m}\right) t, \\ h_{d} & \text { if }\left(u_{m}-c_{m}\right) t<x-a<W t, \\ \text { if } W t<x-a,\end{cases}
$$

where $W$ is the speed of the hydraulic jump given by the Rankine-Hugoniot relation as $W=\frac{h_{m} u_{m}}{h_{m}-h_{r}}$. Note that $c_{m}$ (and thus $h_{m}$ ) is actually the solution of a polynomial equation of degree 6 corresponding to the conservation of the Riemann invariant on the rarefaction wave, and $u_{m}$ is given by the RankineHugoniot relation at the shock wave.

Let us consider initial heights given by $h_{l}=2[\mathrm{~m}]$ and $h_{r}=1[\mathrm{~m}]$. Figure 6 visualizes the profile of the numerical solution, computed on the fine grid of small cells $\mathcal{C}_{h}$ and its comparison with (16) at times $t=0,3$ and 5 [s]. Three mesh sizes and time steps are considered. (The CFL number is smaller than one.) One can see that the numerical simulations again converge to a Navier-Stokes solution 
close to the one predicted by the Saint-Venant equations, and compare well with other 3D simulations like [15]. The front speeds again clearly agree, and the main differences between the Navier-Stokes and the Saint-Venant predictions are again localized close to the discontinuities only. Navier-Stokes equations not only tend to regularize the fronts. But 3D (non-hydrostatic) effects are also clearly seen close to each front. Compared with the exact Saint-Venant solution, an overshoot occurs around the shock wave, together with an oscillation close to the rarefaction wave. Figure 7 shows that the Navier-Stokes solutions (reached in the limit of numerical convergence) includes a significant non-zero vertical component close to the shock wave. These features are not numerical artefacts. They do not completely vanish as the discretization parameters are refined, and they also show up in [15] where the discretization method is quite different.
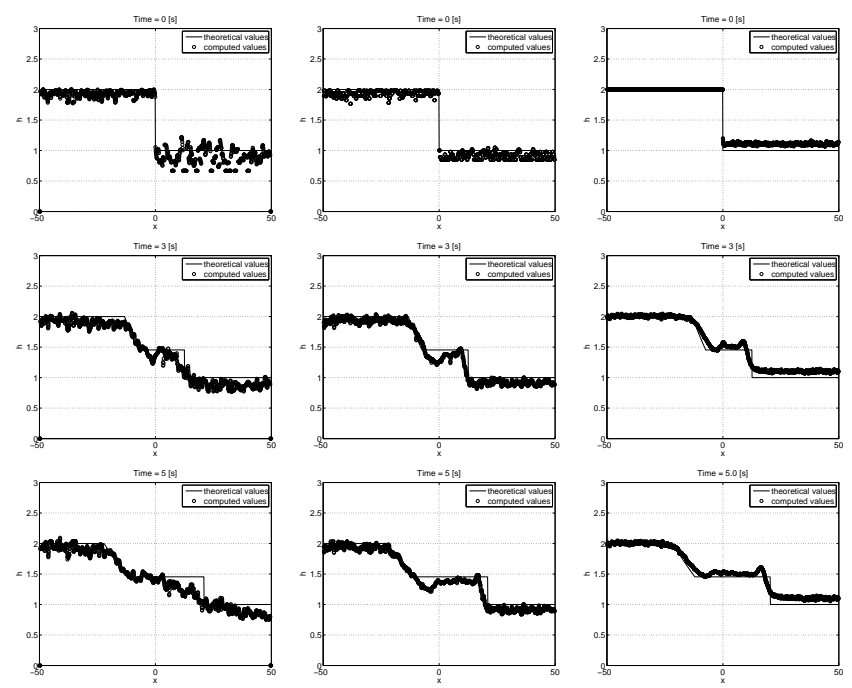

Figure 6: Stoker analytical test case: comparison with analytical solution at times $t=0,3$ and $5[\mathrm{~s}]$. Left: coarse mesh $\left(h=8 \cdot 10^{-2}, H=3 / 5, \tau=0.1\right)$; right: middle mesh $\left(h=6 \cdot 10^{-2}, H=3 / 8, \tau=0.05\right)$; right: fine mesh $(h=$ $\left.4 \cdot 10^{-2}, H=3 / 14, \tau=0.025\right)$. All the grid points on the free surface are represented, including those in the transverse direction.

In order to emphasize the three-dimensional character of the computational solver, let us extend this Stoker test case to a full three-dimensional situation, following [1,22]. The dimensions of the computational domain are $20 \times 20 \times$ $3\left[\mathrm{~m}^{3}\right]$. A cylindrical column of water of height $w_{l}=2.5[\mathrm{~m}]$ and radius $r=$ $2.5[\mathrm{~m}]$ is initially located at the center of the domain. The rest of the domain is filled (wet bed) with water up to an height of $w_{r}=0.5[\mathrm{~m}]$. The column of water is released at time $t=0[\mathrm{~s}]$. The finite element mesh $\mathcal{T}_{H}$ contains 1323720 elements, which corresponds to $H=0.1[\mathrm{~m}]$. The size of the cells in $\mathcal{C}_{h}$ is $h=0.01[\mathrm{~m}]$. The time step is $\tau=0.01[\mathrm{~s}]$. Figure 8 shows the magnitude 

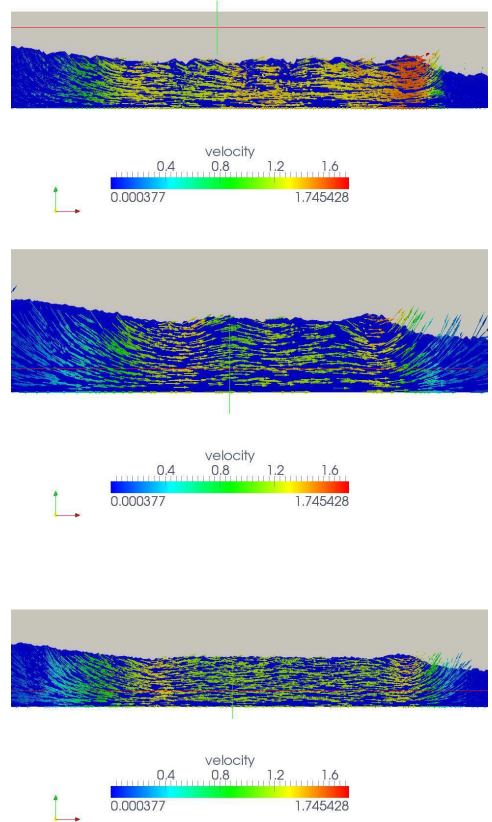

Figure 7: Stoker analytical test case: Visualization of the velocities at time $t=5[\mathrm{~s}]$ next to the liquid front. Top: coarse mesh; middle: middle mesh; bottom: fine mesh. Results magnified by a factor 3 in the vertical direction.

of the water velocity field at different times. The radial invariance of the front is well-preserved, showing that the wave propagation is not influenced by the Cartesian grid $\mathcal{C}_{h}$ for the advection that is aligned with the coordinates axes.
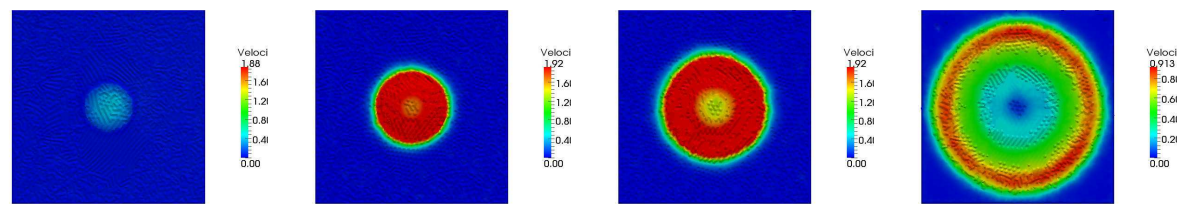

Figure 8: Axisymmetric Stoker test case: visualization of the velocity magnitude of the water surface at times $0,0.5,1.0$ and 2.5 [s] (left to right). 


\subsection{Dam breach over a flat wet bed}

This benchmark problem is a popular test case of a dam break over a wet bed $[4,12,35]$. The dam is partially, instantaneously, broken at time $t=0[\mathrm{~s}]$, in an asymmetric manner, as only a non-central part of the dam is removed - the dam breach.

The computational domain is constructed by vertical extrusion on 20 [m] of a $2 \mathrm{D}$ unstructured mesh of the domain $(0,200) \times(0,200)\left[\mathrm{m}^{2}\right]$. Specific dimensions of the dam can be found, e.g., in [4]. The 3D finite element mesh is composed of 567364 elements and 97146 vertices, for a mesh size $H \simeq 2[\mathrm{~m}]$. The size of the small structured grid of cells is $h=0.5[\mathrm{~m}]$. The time step is $\tau=0.05[\mathrm{~s}]$, implying that the CFL number is close to 0.5 . While slip boundary conditions are still enforced on the lateral walls, we imposed no-slip boundary conditions on the (flat) bottom for this test case, in contrast with the Ritter and Stoker test cases where slip boundary conditions were used. Note that slip and noslip boundary conditions are the two limits between which the whole range of boundary conditions with a friction term like Manning in shallow-water models varies. The location of the liquid at initial time consists of two layers of water at rest (zero initial velocity) with respective heights of $h_{1}=10[\mathrm{~m}]$ (on the left side of the dam) and $h_{2}=5[\mathrm{~m}]$ (on the right side of the dam). At time $t=0[\mathrm{~s}]$, the non-central part of the dam is removed.

Figure 9 visualizes the water height at times $t=0.0,2.5,5.0,7.5,10.0$ and $12.5[\mathrm{~s}]$. Results can be easily compared with those of the literature (in particular in $[4,12]$ and references therein). One can observe that the shape of the contours are very comparable to the existing results of the literature, while the amount of the overshoot of the advancing front is slightly reduced. While the presence of an overshoot at the front is again a manifestation of non-hydrostatic effects, it is significantly reduced here, probably due to the modeling (no-slip boundary conditions) and numerical artifacts inherent to 3D methods (3D diffusion, maybe further increased here because of the rather coarse mesh we use for efficient 3D velocity approximations).

\subsection{Dam break with constriction : comparison with ex- perimental results}

We consider a dam break wave in a channel with a rectangular section, similar to the Ritter test case. The channel has a constriction approximately 12 [m] away from the left extremity of the domain. The experimental conditions, as well as the geometrical quantities, are fully described in [19, 20] and illustrated in Figure 10. The finite element mesh used has 286398 nodes and 1569600 elements, with typical size $H=0.006$ [m]. The structured grid contains 106246000 cells, with typical size $h=0.002[\mathrm{~m}]$. The bottom of the domain is dry and flat, and the water is initially at rest, with height $0.3[\mathrm{~m}]$ ahead of the dam. At initial time, the dam breaks entirely.

This test case is highly non-hydrostatic, since the constriction induces large vertical velocities, as illustrated by snapshots of the numerical solution on the 

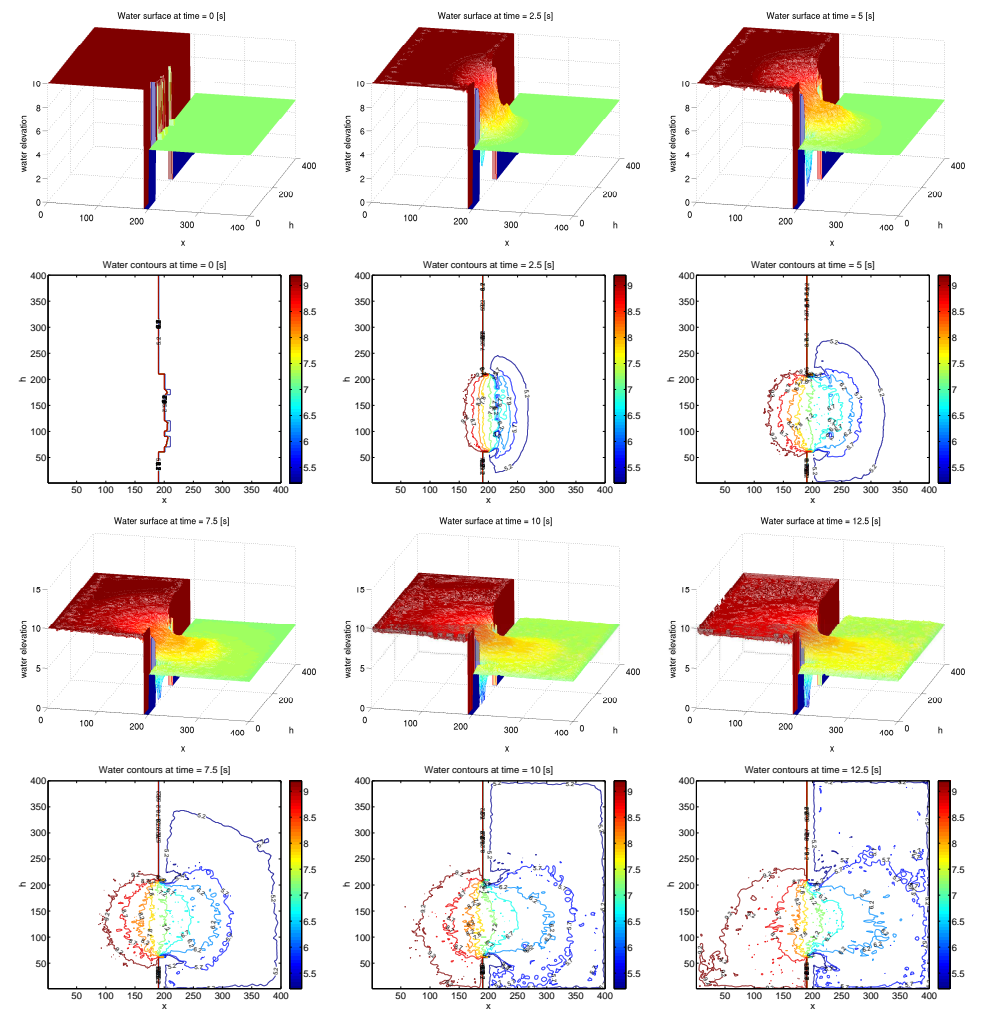

Figure 9: Partial dam break over a flat wet bed at times $t=0.0,2.5,5.0,7.5,10.0$ and 12.5 [s]. Top: water height (in meters); bottom: contours of water height (in meters).

finite element mesh in Figure 11. It is thus a good benchmark to validate the use of 3D simulations and compare with simplified models or experimental data. Measured values of the water level are available at four given points, labeled $S_{1}$ through $S_{4}$, and illustrated in Figure 10. Figure 12 visualizes a comparison of the time evolution of the water level at these four markers with experimental data $[19,20]$. The approximation of the water height is computed on the grid of small cells. Simulation results of the water depth are in agreement with the experimental data for all the four markers. Actually, results at the third marker $S_{3}$ are significantly better than those given, e.g., in [19] when using an hydrostatic simplified model. On the other hand, results at the fourth marker $S_{4}$ are less accurate due to the very shallow behavior of the water after the constriction (meaning shallow water-like models are more adapted here).

Remark that, the narrower the constriction, the slowlier the liquid goes into the channel, as the backward wave becomes stronger. Numerical investigations have shown precisely that, if the channel is $10[\mathrm{~cm}]$ wide instead of $25[\mathrm{~cm}]$, the 
behavior of the water level for marker $S_{1}$ is unchanged, but the water does not reach the marker $S_{4}$ in the time interval considered.

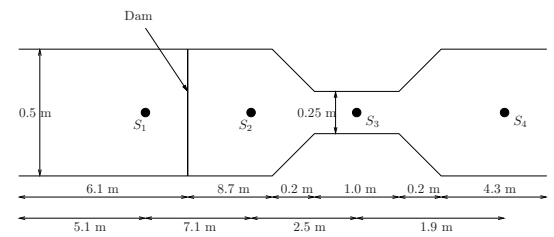

Figure 10: Dam break with constriction. Notation, dimensions and location of the markers.

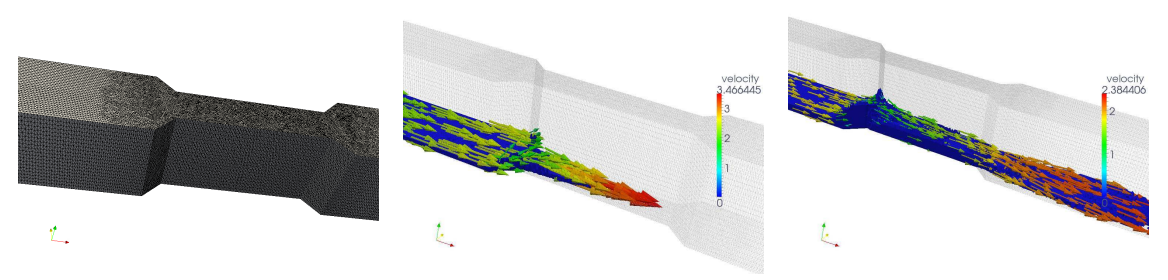

Figure 11: Dam break with constriction; visualization of the mesh near the constriction and snapshots of the solution at times $t=0.4,0.6[\mathrm{~s}]$.
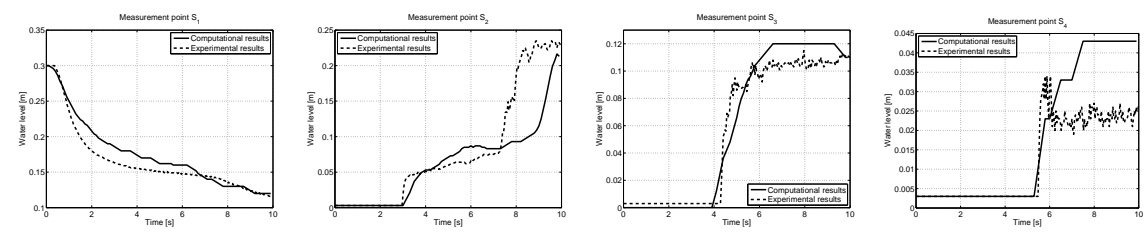

Figure 12: Dam break with constriction. Time evolution of the water height at the markers $S_{1}, S_{2}, S_{3}$ and $S_{4}$, and comparison with experimental data [19].

\subsection{Malpasset dam break}

The Malpasset dam break is a real-life test case. The Malpasset dam was located approximately 12000 [m] upstream of Frejus on the French Riviera. The maximum reservoir capacity was meant to be $55106\left[\mathrm{~m}^{3}\right]$. The dam failed explosively on December 2, 1959, and the flood wave ran along the valley to Frejus. The evolution of the water front and water height has been well-documented via data collection and measurement, or reproduction with a physical model or computations. 
The breakage of the Malpasset dam has been widely treated in the literature, see, e.g., $[2,13,17,18]$. This test case has also been a benchmark model for Electricité de France (EDF) for several years, in order to validate simplified 1D or 2D models based on shallow water equations. 3D simulations of the Malpasset dam break are less frequent and can be found, for instance, in [3, 21].

The Malpasset test-case allows one to compare a full 3D model with simplified 1D or 2D models with a view to reproducing experimental results. First, the friction at bottom in Navier-Stokes equations can be simply modelled by a no-slip boundary condition (the singular limit of the Navier friction boundary condition) and does not necessarily require tuning a parameter like in SaintVenant equations. Second, the non-hydrostatic features of the flow in the presence of a non-trivial topography can be discussed. To the best of our knowledge, only qualitative results of the Malpasset dam break have been presented when using 3D numerical simulations. Here, we aim at quantifying some effects of the 3D approach.

The 3D computational domain is constructed by extrusion. The 2D map of the topography has been digitized from ancient topographical maps (see [17] and references therein). The overall dimensions of the domain are $17500[\mathrm{~m}]$ $\times 9000[\mathrm{~m}]$. Elevation of the valley ranges from $-20[\mathrm{~m}]$ (below sea level) to +100 [m] (above sea level); this latter value is an estimation of the initial free surface elevation in the reservoir. The 2D mesh contains 13541 points and 39541 triangles. It is illustrated in Figure 13 (top), as actually available in the public domain. This mesh is then extruded over 10 layers of prisms, each of them cut into six tetrahedra, to form a 3D finite element mesh $\mathcal{T}_{H}$ of 311443 vertices and 1716000 elements, with resolution $H=5$ [m]. The cell size of the structured mesh is $h=2[\mathrm{~m}]$.

The dam structure is following a straight line between the points of coordinates: $(4701[\mathrm{~m}], 4143[\mathrm{~m}])$ and $(4655[\mathrm{~m}], 4392[\mathrm{~m}])$. The reservoir level is located behind the dam, at a constant water level equal to $100[\mathrm{~m}]$. The level of the Mediterranean sea is constant and equal to zero. Initial conditions are also represented in Figure 13 (bottom). The remaining part of the bottom of the domain is dry. At time $t=0$, the dam is completely and instantaneously removed; the water therefore flows down the valley. Recall that no-slip boundary conditions are enforced on the bottom topography (unlike published results we do not calibrate any friction coefficient). Several points of given coordinates (gauge points) have been measured thanks to a reduced-scale physical model. They can be used for comparison with the numerical results. The coordinates of these particular points can be found, e.g. in [17, 21].

Figure 14 shows snapshots of the solution at times $t=0,100,200,300,400$ and $500[\mathrm{~s}]$, together with a visualization of the velocity field. Each time step, corresponding to one second of simulation, takes approx. 10 minutes of $\mathrm{CPU}$ time.

Figure 15 shows a comparison between the numerical results and physical results obtained with the reduced scale model or computational results using simplified models [21]. The comparison focuses on the maximal water height (left) and the arrival time of the first water wave (right) at the gauge points. 


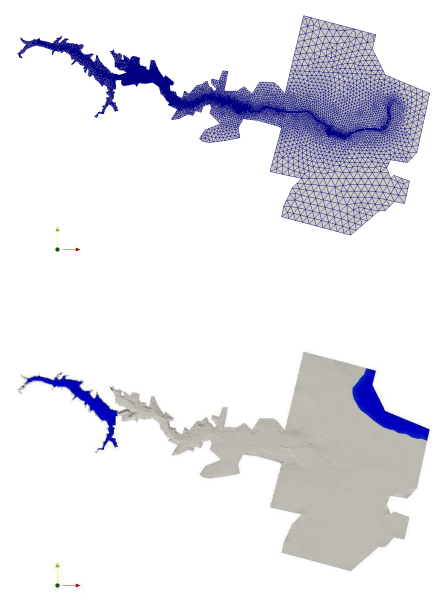

Figure 13: Malpasset test case: Two-dimensional mesh of the topography (top) and initial position of the liquid - the lake behind the dam and the sea (bottom).

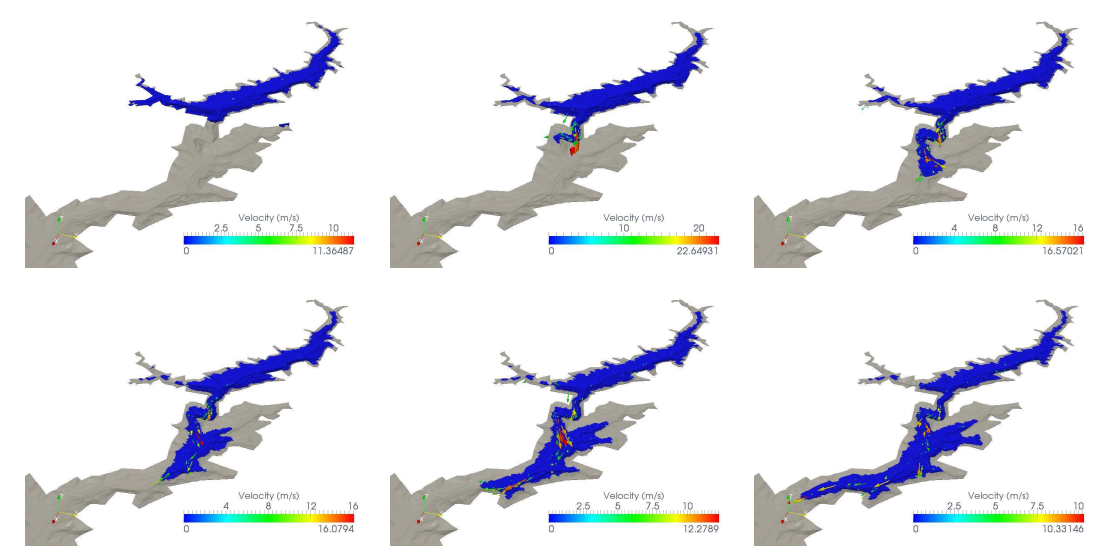

Figure 14: Malpasset dam break: snapshots of the 3D solution at times $t=$ $0,100,200,300,400$ and 500 [s].

Figure 15 (right) shows that the computed arrival times of the water wave at these given points is larger than the ones of the physical model. This means that the wave calculated with the 3D numerical simulation actually travels slowlier than the physical one. This is not surprising and is a consequence of i) the inherent numerical diffusion of a three-dimensional model due to a relatively 
large mesh size; ii) the no-slip boundary conditions on the topography that slow the water evolution.

On the other hand, Figure 15 (left) shows the maximal water level at these gauge points, when the overshoot observed for instance in Figure 6 for the Stoker test case is smoothed. We observe that numerical results are rather close to existing results. Little oscillations on the water levels happen usually on one time step. They originate mostly at the forefront of a shock wave, when the topography of the bedrock varies quite fast below the markers or when the markers are physically located at the intersection of valleys where interacting waves may amplify the shock. These oscillations are inherent to the $3 \mathrm{D}$ character of the model, and reveal some local non-hydrostatic features that, compared with the Stoker test case, are amplified by the topography.
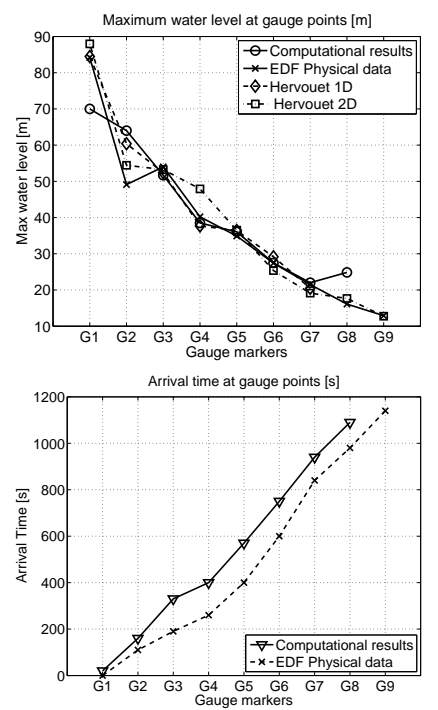

Figure 15: Malpasset dam break: maximum water level (top) and wave arrival times (bottom) at the gauge points. Comparison between numerical results, results from [21] and results from the reduced-scale physical model.

\subsection{Grande-Dixence dam break}

The last numerical experiment tackles the hypothetical dam break of the GrandeDixence dam located in Switzerland, and the resulting flood in the valley $\mathrm{Val}$ d'Hérens. It illustrates the capability to perform large-scale simulations in reallife topographical geometries, and the interest in visualizing $3 \mathrm{D}$ simulations, but has no validation purposes.

The Grande-Dixence dam is the tallest dam in Switzerland. Opened in 1965, it is $285[\mathrm{~m}]$ high and the lake created behind the dam (Lac des Dix) contains 
$400\left[\mathrm{mio} \mathrm{m}^{3}\right]$ of water. It is located at the top of a $30000[\mathrm{~m}]$ long valley leading to the river Rhone and directly above the city of Sion.

The computational domain is constructed as follows. A two-dimensional elevation map is obtained from Swiss topographical data. The resolution of the structured two-dimensional mesh is $25[\mathrm{~m}]$. The 3D finite element mesh is generated by extrusion of the 2D map on 10 layers of prisms, split into six tetrahedra each, leading to a finite element mesh composed of 13876525 elements and 2057005 vertices with resolution $H=50$ [m]. The computational domain is thus $5750[\mathrm{~m}]$ wide, $28900[\mathrm{~m}]$ long and $400[\mathrm{~m}]$ high. On the other hand, the structured grid of small cells has a resolution of $h=10[\mathrm{~m}]$. No-slip boundary conditions are imposed on the bottom topography. The dam of height $285[\mathrm{~m}]$ is initially assumed filled with $400\left[\mathrm{mio} \mathrm{m}^{3}\right]$ of water at rest.

Figure 16 illustrates the location of the liquid front at several times of the simulation and the flooding areas in the valley. Figure 17 illustrates snapshots of the water domain colored according to the instantaneous liquid velocity, which ranges from 0 to $120[\mathrm{~m} / \mathrm{s}]$. Finally, Figure 18 illustrates the water height at several time steps. The water height ranges from 0 to 200 meters outside the initial lake reservoir.
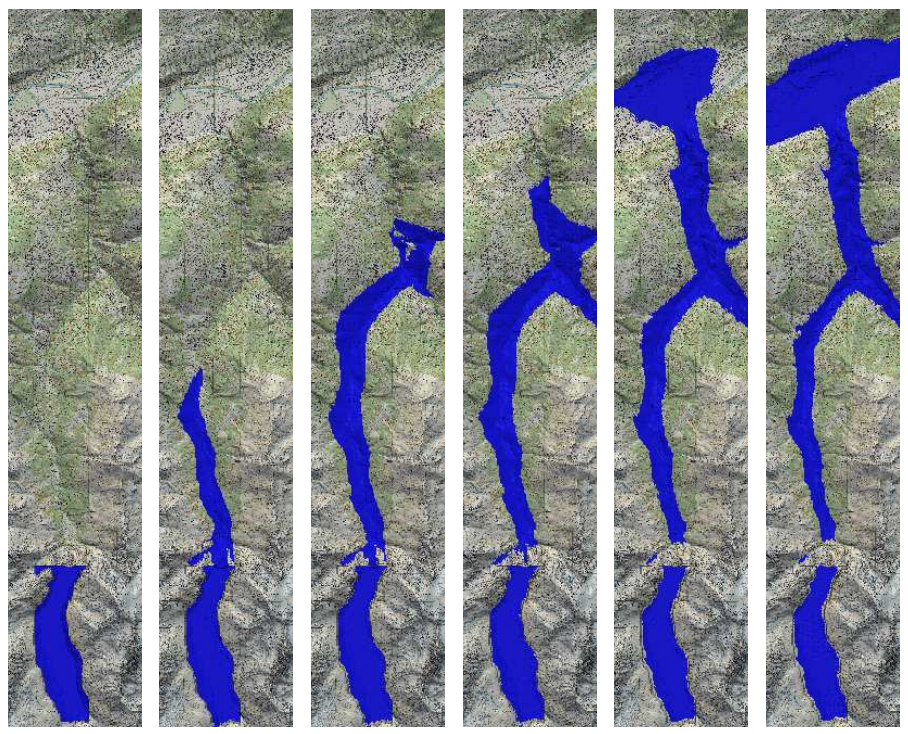

Figure 16: Numerical solution of the Dixence dam break. Snapshots of the liquid domain (top view) at times $t=0,2,4,6,8,10$ [min]. 

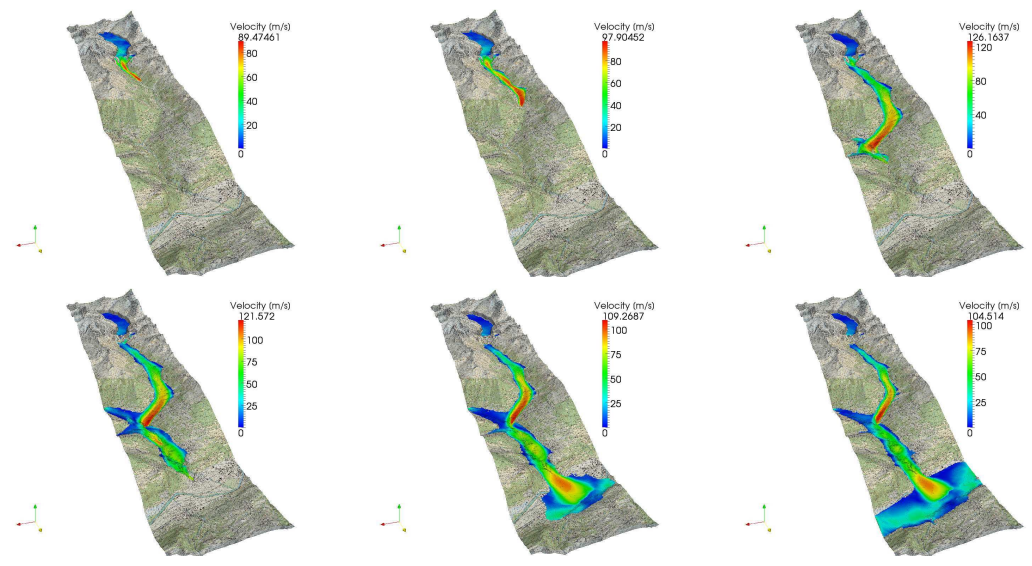

Figure 17: Numerical solution of the Dixence dam break. Snapshots of the liquid domain with fluid velocity at times $t=1,2,4,6,8,10$ [min].
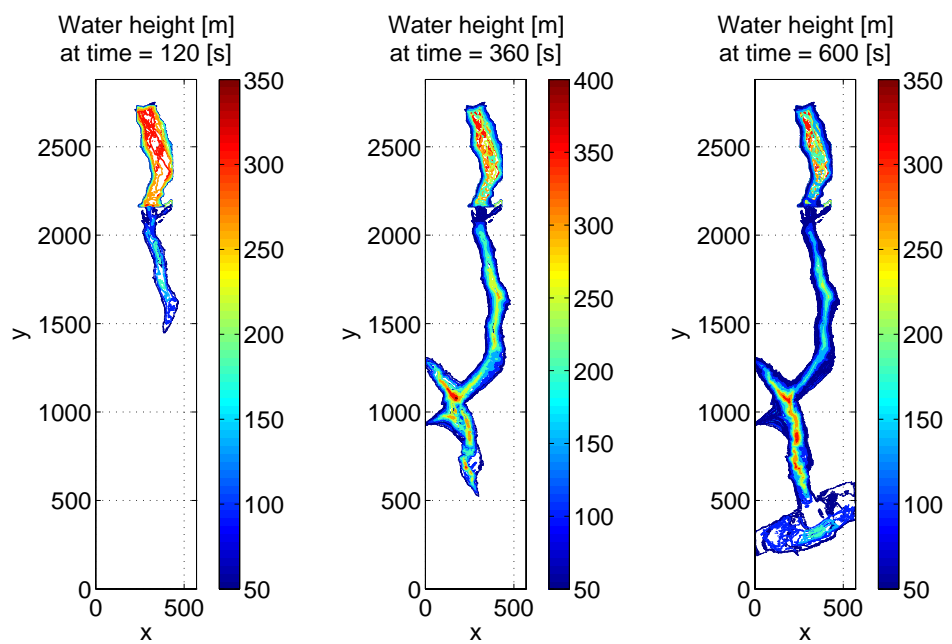

Figure 18: Numerical solution of the Dixence dam break. Snapshots of the water level (top view) at times $t=0,2,4,6,8,10$ [min].

\section{Conclusions and Further Comments}

A numerical method for the simulation of full three-dimensional free-surface flows has been presented. The proposed computational framework has been successful in solving a variety of test cases (from simple benchmarks to real-life situations) with a view to simulating dam breaks. In particular, the numerical 
results show the capability of a full 3D model based on the Navier-Stokes equations at satisfactorily capturing the hyperbolic behavior of water waves while showing non-hydrostatic features that are not present in most reduced models. The non-hydrostatic features of the full 3D modeling of dam breaks flows could be investigated on the basis of these numerical experiments.

Such computational results can thus be very useful to policy makers when delimiting flooding areas and drawing flooding maps, as well as to the hydraulic engineers that are constantly looking for more accurate numerical results of dam break flows (an everlasting major challenge in the hydraulic engineering community). Further work includes the application of the numerical method presented in this article to other practical problems in hydraulic situations, such as the modeling and assessment of spillways discharge capacity or that of the sediment transport.

\section{Acknowledgements}

This work was started while S. Boyaval was a MATHICSE-ASN academic host at EPFL. The authors thank Marco Picasso and Jacques Rappaz (EPFL) for fruitful discussions. S. Boyaval would like to thank Riadh Ata (EDF R\&D), Marie-Odile Bristeau (INRIA) and Nicole Goutal (EDF R\&D) for their numerous advice and for providing the $2 \mathrm{D}$ mesh for the Malpasset test case. A. Caboussat is partially funded by the Swiss national science foundation (grant number 200021_143470).

\section{References}

[1] F. Alcrudo and P. Garcia-Navarro. A high-resolution Godunov-type scheme in finite volumes for the two-dimensional shallow-water equations. Int. J. Numer. Meth. Fluids, 16:489-505, 1993.

[2] F. Alcrudo and E. Gil. The Malpasset dam break case study. In Proceedings of the Fourth CADAM Meeting, pages 95-109, Zaragoza, Spain, 1999.

[3] E. Audusse, M. O. Bristeau, and A. Decoene. 3D free surface flows simulations using a multilayer Saint-Venant model. Comparisons with NavierStokes solutions. In Alfredo Berumdez de Castro, Dolores Gomez, Peregrina Quintela, and Pilar Salgado, editors, Numerical Mathematics and Advanced Applications, pages 181-189. Springer Berlin Heidelberg, 2006.

[4] C. Biscarini, S. Di Francesco, and P. Manciola. CFD modelling approach for dam break flow studies. Hydrol. Earth. Syst. Sci., 14:705-718, 2010.

[5] M.-O. Bristeau, N. Goutal, and J. Sainte-Marie. Numerical simulations of a non-hydrostatic shallow water model. Computers $\&$ Fluids, 47(1):51 64, 2011. 
[6] A. Caboussat. A numerical method for the simulation of free surface flows with surface tension. Computers and Fluids, 35(10):1205-1216, 2006.

[7] A. Caboussat, P. Clausen, and J. Rappaz. Numerical simulation of twophase flow with interface tracking by adaptive Eulerian grid subdivision. Math. Comput. Modelling, 55:490-504, 2012.

[8] A. Caboussat, M. Picasso, and J. Rappaz. Numerical simulation of free surface incompressible liquid flows surrounded by compressible gas. J. Comput. Phys., 203(2):626-649, 2005.

[9] V. Casulli and G. S. Stelling. Semi-implicit subgrid modelling of threedimensional free-surface flows. Int. J. Numer. Meth. Fluids., 67:441-449, 2011.

[10] V. Casulli and P. Zanolli. Comparing analytical and numerical solution of nonlinear two and three-dimensional hydrostatic flows. Int. J. Numer. Meth. Fluids., 53:1049-1062, 2007.

[11] H. Chanson. Application of the method of characteristics to the dam break wave problem. J. Hydr. Research, 47(1):41-49, 2009.

[12] R. J. Fennema and M. H. Chaudhury. Explicit methods for 2-D transient free-surface flows. J. Hydr. Engrg, 116(8):1013-1034, 1990.

[13] S. Soares Frazao, F. Alcrudo, and N. Goutal. Dam-break test cases summary. In Proceedings of the Fourth CADAM Meeting, pages 9-25, Zaragoza, Spain, 1999.

[14] D. L. George. Adaptive finite volume methods with well-balanced Riemman solvers for modeling floods in rugged terrain: Application to the Malpasset dam-break flood (France, 1959). Int. J. Numer. Meth. Fluids, 66(8):10001018, 2011.

[15] J.-F. Gerbeau and B. Perthame. Derivation of viscous Saint-Venant system for laminar shallow water; numerical validation. Discrete and Continuous Dynamical Systems, Ser. B, 1(1):89-102, 2001.

[16] R. Glowinski. Finite Element Method For Incompressible Viscous Flow, volume IX of Handbook of Numerical Analysis (P.G. Ciarlet, J.L. Lions eds), pages 3-1176. Elsevier, Amsterdam, 2003.

[17] N. Goutal. The Malpasset dam failure. An overview and test case definition. In Proceedings of the Fourth CADAM Meeting, pages 1-8, Zaragoza, Spain, 1999.

[18] N. Goutal. Presentation of 1D and 2D simulations of Malpasset dam break wave propagation. In Proceedings of the Fourth CADAM Meeting, pages 119-130, Zaragoza, Spain, 1999. 
[19] N. Goutal and J. Sainte-Marie. A kinetic interpretation of the sectionaveraged Saint-Venant system for natural river hydraulics. Int. J. Numer. Meth. Fluids, 67:914-938, 2011.

[20] N. Goutal and M. Slydowski. Validation d'un schéma volumes finis pour les équations de Saint-Venant bidimensionnelles. Technical report, Département Laboratoire National d'Hydraulique, EDF, 1998.

[21] J.-M. Hervouet. Hydrodynamics of Free Surface Flows: Modelling with the Finite Element Method. Wiley, London, 2007.

[22] Y. Loukili and A. Soulaïmani. Numerical tracking of shallow water waves by the unstructured finite volume WAF approximation. Int. J. Computational Methods in Engineering Science and Mechanics, 8:1-14, 2007.

[23] A. De Maio, F. Savi, and L. Sclafani. Three-dimensional mathematical simulation of dambreak flow. In Proceeding of IASTED conferences Environmental Modelling and Simulation, St. Thomas, US Virgin Island, 2004.

[24] F. Marche. Derivation of a new two-dimensional viscous shallow water model with varying topography, bottom friction and capillary effects. $E u$ ropean Journal of Mechanics-B/Fluids, 26(1):49-63, 2007.

[25] V. Maronnier, M. Picasso, and J. Rappaz. Numerical simulation of three dimensional free surface flows. Int. J. Num. Meth. Fluids, 42(7):697-716, 2003.

[26] M. Mohammadi. Boundary shear stress around bridge piers. Am. J. Appl. Sci., 5(11):1546-1550, 2008.

[27] N. Nagata, T. Hosoda, T. Nakato, and Y. Muramoto. Three-dimensional numerical model for flow and bed deformation around river hydraulic. $J$. Hydraul. Eng., 131:1074-1087, 2005.

[28] W.F. Noh and P. Woodward. SLIC (Simple Line Interface Calculation), volume 59 of Lectures Notes in Physics, pages 330-340. Springer-Verlag, 1976.

[29] I. R. Park, K. S. Kim, J. Kim, and S. H. Van. A volume-of-fluid method for incompressible free surface flows. Int. J. Numer. Meth. Fluids., 61:13311362, 2009.

[30] A. Ritter. Die fortpflanzung der wasserwellen. Zeitschrift des Vereins Deutscher Ingenieure, 36:947-954, 1892.

[31] M. Shashkov and B. Wendroff. The repair paradigm and application to conservation laws. J. Comput. Phys., 198(1):265-277, 2004. 
[32] Y. Shi and K. D. Nguyen. A projection method-based model for dam- and dyke-breaks flows unsing an unstructured finite-volume technique: Applications to the Malpasset dam break (France) and to the flood diversion in the Red River basin (Vietnam). Int. J. Numer. Meth. Fluids, 56(8):1505-1512, 2008.

[33] T. Shigematsu, P. L.-F. Liu, and K. Oda. Numerical modeling of the initial stages of dam-break waves. J. Hydr. Research, 42(2):183-195, 2004.

[34] J. J. Stoker. Water Waves: The Mathematical Theory with Applications. Interscience, London, 1957.

[35] A. Valiani, V. Caleffi, and A. Zanni. Case study: Malpasset dam break simulation using a two-dimensional finite volume method. J. Hydr. Engrg, 128(5):460-472, 2002.

[36] J. Wang, R. Zou, and D. Wan. Numerical simulation of 3D dam-break flow by FEM-level set method. In Proceedings of the Twenty-second (2012) International Offshore and Polar Engineering Conference, pages 1080-1081, 2012 . 